\title{
Combining ultrahigh-resolution ion-mobility spectrometry with cryo- genic IR spectroscopy for the analysis of glycan mixtures
}

\author{
Ahmed Ben Faleh, Stephan Warnke, and Thomas R. Rizzo* \\ Laboratoire de Chimie Physique Moléculaire, École Polytechnique Fédérale de Lausanne, EPFL SB ISIC LCPM, Station 6, \\ CH-1015 Lausanne, Switzerland
}

KEYWORDS: glycans, carbohydrates, ion mobility spectrometry, ion spectroscopy, mass spectrometry

\begin{abstract}
The isomeric complexity of glycans make their analysis by traditional techniques particularly challenging. While the recent combination of ion mobility spectrometry (IMS) with cryogenic IR spectroscopy has demonstrated promise as a new technique for glycan analysis, this approach has been limited by the modest resolution of the ion mobility stage. In this work we report results from a newly developed instrument that combines ultrahigh-resolution IMS with cryogenic IR spectroscopy for glycan analysis. This apparatus makes use of the recent development in travelling-wave IMS called structures for lossless ion manipulation (SLIM). The IMS stage allows the selection of glycan isomers that differ in collisional cross section by as little as $0.2 \%$ before injecting them into a cryogenic ion trap for IR spectral analysis. We compare our results to those using drift-tube IMS and highlight the advantages of the substantial increase in resolution. Application of this approach to glycan mixtures demonstrates our ability to isolate individual components, measure a cryogenic IR spectrum, and identify them using a spectroscopic database.
\end{abstract}

\section{INTRODUCTION}

It is difficult to overstate the importance of glycans in biological systems. ${ }^{1-3}$ Nearly every cell of every organism is coated with a layer of glycans that plays a key role in cell-to-cell recognition and signaling. Glycans govern the interaction of cells with bacteria and viruses and are central to molecular recognition, immune response, and inflammation. ${ }^{4}$ Despite their importance, glycans are extremely difficult to analyze because of their isomeric complexity. Unlike linear sequences of monomers with distinct mass as found in proteins, many of the monosaccharide building blocks of glycans are isomeric, differing only in the stereochemistry of the asymmetric carbon atoms. In addition, the glycosidic bond linking these monosaccharides involves a stereogenic carbon, leading to isomeric $\alpha$ and $\beta$ anomers. Moreover, glycosidic bonds can have different attachment points, leading to different regioisomers. Finally, the ability of a monosaccharide unit to support multiple glycosidic bonds can result in the formation of branched structures that are isomeric with the corresponding linear chains of the same monosaccharide content. Because these various types of isomerism are present simultaneously, there is a vast number of possible glycan isomers, ${ }^{5}$ and many of the powerful tools used to sequence proteins are not able to distinguish them.

The speed and sensitivity of mass spectrometry (MS) make it particularly attractive as a tool for glycan analysis. The most detailed structural information comes from the use of tandem MS techniques, ${ }^{6-7}$ and these have employed a variety of different dissociation methods to generate the cross-ring fragments needed to determine linkage positions and stereochemistry. ${ }^{8-14}$ While permethylation of the free hydroxyl groups prior to fragmentation ${ }^{12,15}$ helps to reveal the locations of the glycosidic bonds, even MS-MS cannot distinguish all of the various glycan isomers. Several studies have shown that the combination of ion mobility and mass spectrometry (IMS-MS) can often resolve glycan isomers that are indistinguishable by MS alone. ${ }^{16-23} \mathrm{~A}$ particularly promising approach is to combine tandem MS with ion mobility, using both the mass of the fragments and their collision cross section to help reconstruct the primary structure of the parent glycan. ${ }^{24-26}$ Nevertheless, IMS is blind to many of the subtle structural details that distinguish isomeric glycans. ${ }^{18}$

One other possibility is to add a spectroscopic dimension to MS to achieve further isomer discrimination, since spectroscopic fingerprints can be extremely sensitive to the slightest differences in structure. Several groups have successfully combined MS with infrared multiple-photon dissociation (IRMPD) spectroscopy for identification of small glycans, ${ }^{27-32}$ but the absorption bands in the room temperature IRMPD spectra are often too broad to uniquely identify isomeric disaccharides in a mixture. In recent work, Mucha et al. ${ }^{33}$ used a free-electron laser to obtain spectroscopic fingerprints of oligosaccharides cooled in liquid helium droplets and demonstrated that sufficiently resolved spectra could indeed distinguish the various types of isomerism, albeit with an extremely complex instrument that would be impractical as a broadly used tool.

Recently, IRMPD spectroscopy has been combined with tandem MS and ion mobility to determine the structure of small glycans. ${ }^{30}$ In this case, information from separately performed IR-MS/MS and IMS-MS/MS experiments on the same systems are used together. ${ }^{30}$ Results from this work are particularly significant in that they suggest that stereochemistry of the glycosidic bond is retained in the C-fragments of cationized disaccharides. ${ }^{34}$ More recently, the group of Compagnon has combined HPLC and IRMPD spectroscopy to measure spectra of the two anomeric forms of glucosamine separately. ${ }^{35}$

In a series of proof-of-principle experiments, ${ }^{36-38}$ we have recently combined ion mobility spectrometry in a $2 \mathrm{~m}$ drift tube 
with messenger-tagging infrared spectroscopy in a cryogenic ion trap coupled to a TOF-MS to identify and characterize glycans. Our application of this approach to a set of isomeric disaccharides ${ }^{37-38}$ as well as series of human milk oligosaccharides $^{36}$ clearly demonstrate that a low-temperature vibrational spectrum can easily distinguish the subtlest differences between glycan isomers. Although we succeeded in characterizing different isomers, the IMS arrival-time distributions (ATDs) for the isomeric species were very similar, making it difficult to separate them. While a spectral deconvolution could in principle determine the composition of a complex mixture, an increase in the resolving power of the IMS stage that would allow us to separate isomers with extremely small differences in CCS would greatly simplify the deconvolution procedure. A promising direction in the field of ultrahigh-resolution IMS has been the development by Smith and co-workers of what they call Structures for Lossless Ion Manipulations (SLIM), ${ }^{39-40}$ which is a type of travelling wave ion mobility using a "sandwich" of printed circuit boards that combine RF and DC potentials. Recent work from Nagy et al. ${ }^{41}$ has demonstrated the possibility of using SLIM to separate different conformers of a single disaccharide, speculated to be its $\alpha$ and $\beta$ anomers.

In this work, we describe and characterize our newly developed approach combining ultrahigh-resolution, SLIM-based, IMS-MS with messenger-tagging, cryogenic IR spectroscopy for the characterization of glycans. We compare our results to our previously published drift-tube IMS results and demonstrate the simplification that arises from increasing the IMS resolution by more than a factor of 6 . As a proof of principle, we use this method to identify disaccharide isomers from mixtures. Finally, we apply our method to a pair of human milk oligosaccharides, demonstrating that it can identify the composition of mixtures of biologically relevant components.

\section{EXPERIMENTAL APPROACH}

Samples. Oligosaccharide samples were purchased from Dextra (UK) and Carbosynth (UK) and used without further purification. For nano-electrospray ionization (nESI), the samples were diluted in 50/50 water/methanol or water/acetonitrile at a concentration of $100 \mu \mathrm{M}$. All our measurements were made on the sodiated species, and thus we add 1.5 equivalents of $\mathrm{NaCl}$ to enhance their formation. Because our goal is to identify the isomeric form of glycans and not their 3-dimensional structure, the fact that we study the sodiated species has no consequence, as long as the database to which we compare is constructed for sodiated glycans.

Instrument. We perform our experiments on a home-built, ion-mobility mass spectrometer, shown schematically in Fig. $1(\mathrm{a})$, which is equipped with a cryogenic ion trap ${ }^{42}$ and a timeof-flight (TOF) mass spectrometer. The major difference between this machine and that used in our previous work ${ }^{43-45}$ is the addition of a travelling-wave ion mobility device based on SLIM $^{39-40}$ which was appended to the opposing arm of the electrostatic bender that turns the ions to the cryogenic ion trap.

Ions produced by nano-electrospray were transferred into the instrument through a flared stainless-steel capillary ${ }^{46}$ and stored in the hourglass section of a commercial dual ion-funnel trap assembly (MassTech, USA). ${ }^{47}$ From there, ion pulses $120 \mu \mathrm{s}$ in duration are released into the ion-mobility section, where they are separated based on their respective collision cross sections (CCS). They are then directed through differential pumping stages using ring-electrode ion guides and hexapoles. Ions of a particular drift time can be selected by electrostatically deflecting unwanted ions using a steering-lens assembly prior to the electrostatic ion bender. After $\mathrm{m} / \mathrm{z}$ selection in a quadrupole mass filter, the ions enter the cryogenic ion trap (40 K) where they are stored and cooled through collisions with a cold buffer gas composed of helium and nitrogen in a 90:10 mixture. Nitrogen molecules condense on the internally cold ions, which can be observed as a shift in mass in subsequent TOF analysis. In order to record an IR spectrum, the content of the ion trap is irradiated by a single pulse $(\sim 10 \mathrm{~mJ})$ from an IR OPO system. When the light is resonant with a molecular vibration, the absorption of a single photon and subsequent redistribution of vibrational energy causes the weakly bound $\mathrm{N}_{2}$ molecule to boil off. An IR spectrum of the $\mathrm{m} / \mathrm{z}$ - and size-selected ions is generated by monitoring the wavelength-dependent ion signal of the $\mathrm{N}_{2}$ tagged species in the TOF mass spectrum. Spectral data points are acquired in $1 \mathrm{~cm}^{-1}$ increments by averaging signal from 10 filling events at a repetition rate of $10 \mathrm{~Hz}$, which is determined by our OPO. For the wavenumber range presented here, a single scan requires six to eight minutes to complete, and our final data represents the average of two such scans. An ionmobility arrival time distribution (ATD) can be recorded by inserting a channeltron electron multiplier to monitor the timedependent ion current of $\mathrm{m} / \mathrm{z}$-selected ions after they pass through the quadrupole MS.

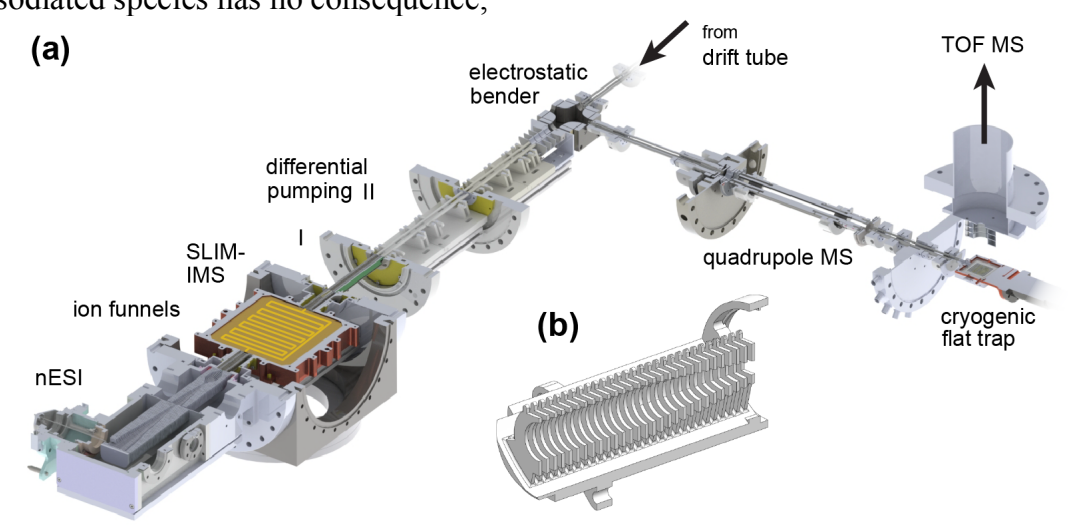

Figure 1 (a) Overview of the newly developed instrument combining ultrahigh resolution SLIM IMS with cryogenic messenger-tagging IR spectroscopy. (b) Cross-sectional view of one of the 3D-printed ion guides showing the morphed inner geometry of the ring electrodes. 
To achieve ultrahigh-resolution ion-mobility separation, we use SLIM-based travelling-wave ion mobility spectrometry (TW-IMS) developed by Smith and coworkers. ${ }^{39-40}$ A pair of mirrored printed circuit boards (PCBs) produce confining RF and DC fields as well as a travelling square-well potential (Fig. S1(a), Supporting Information) $)^{39,48}$ that propels the ions through a drift gas. The potential is defined by a repeating sequence of eight electrodes to which we apply a DC voltage in a binary fashion: four pads receive a positive $\mathrm{DC}$ voltage while the following four are kept at $0 \mathrm{~V}$. This situation can be represented by a binary sequence of the type 11110000 (Fig. S1(a)). The potential wave then propagates by shifting this sequence one bit at a time to 01111000 and so on. Smith and coworkers have demonstrated that these fields can be used to propel ions around corners, and thus one can lead them along a serpentine path $^{48}$ that covers a large fraction of the surface area of the printed circuit board.

In our implementation of this technique, illustrated in Fig. S1(b) (Supporting Information), we use PCBs $15 \times 15 \mathrm{~cm}$ on edge placed $2.75 \mathrm{~mm}$ apart to achieve a single-pass ion path of approximately $1.8 \mathrm{~m}$. Moreover, we incorporate an electrostatic switch $^{49}$ at the end of the drift path either to send ions towards the cryogenic ion trap or to cycle them multiple times through the same track, thereby increasing the effective path length and hence the resolving power, which should scale with $\sqrt{n} .{ }^{50}$

For each type of molecule, the optimum TW amplitude and speed for ion mobility separation are determined empirically. Typical IMS parameters for the here-reported experiments were: RF frequency $880 \mathrm{kHz}$; RF amplitude $100 \mathrm{~V}_{\mathrm{pp}}$; TW amplitude $27 \mathrm{~V}$; TW speed $600 \mathrm{~m} / \mathrm{s}$; He buffer gas pressure 3 mbar.

The ion guides and funnels used in the ion-mobility region of the setup consist of 3D-printed material (Formlabs, USA) that was subsequently coated with nickel to provide a robust, singlepiece solution with electrically conductive surfaces. ${ }^{51}$ The guides preceding and following the SLIM device consist of a ring-electrode design with a morphed inner shape to match both the rectangular geometry of the SLIM structure and the circular geometry of the conductance limits, as shown in Fig. 1(b). Similarly, a symmetric, 3D-printed ring-electrode guide, as well as two hexapoles of conventional geometry, direct the ions through the two subsequent differentially pumped regions with pressures $10^{-2}$ mbar and $10^{-5} \mathrm{mbar}$, respectively.

(a)

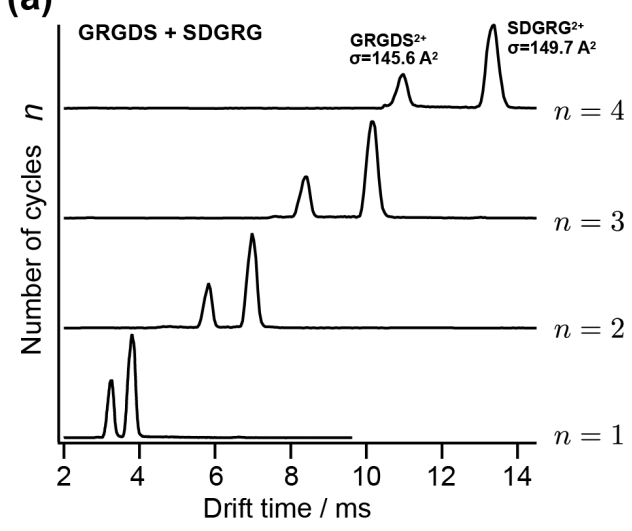

\section{RESULTS AND DISCUSSION}

Performance of the SLIM module. Ions that complete a cycle on the serpentine drift path of the SLIM module can be either extracted from the SLIM region and sent towards the cryogenic ion trap or routed back to the beginning of the drift section to complete another ion-mobility separation cycle until the desired resolving power is reached. We tested the capability of this device to separate ions of different collision cross section using the isomeric peptides GRGDS and SDGRG, which, in their doubly protonated form, differ in CCS by approximately $2.8 \%{ }^{52}$ An arrival time distribution of an electrosprayed mixture of the two peptides completing a single separation cycle on the SLIM device is shown in the lower panel of Fig. 2(a).

After a single pass, the separation between the two species is approximately equivalent to that achieved with a $2 \mathrm{~m}$ drift tube instrument, ${ }^{52}$ and it continues to increase as the number of separation cycles is increased (other ATDs in Fig. 2(a)). The resolution of a travelling wave IMS instrument cannot be directly deduced from the drift-time information alone, because the relationship between the collision cross section and the drift time is not straightforward to deduce.$^{50}$ Instead, we quantify the resolution of our device by calibrating the drift time with the previously published CCS values of $\operatorname{GRGDS}^{2+}\left(145.6 \AA^{2}\right)$ and $\operatorname{SDGRG}^{2+}\left(149.7 \AA^{2}\right)$, assuming a linear relation over this small range. Figure 2(b) shows the resulting resolution $R$ for these two peptides as a function of the number of cycles $n$, determined using the peak-width definition $R=\Omega / \Delta \Omega$, where $\Omega$ and $\Delta \Omega$ are the CCS and the peak width on the CCS axis, respectively. The expected $\sqrt{n L}$ dependency of $R$ is confirmed by the good fit (black line) of the function $f(n)=a+b \sqrt{n}$ to the data in Fig. 2(b). The instrument reaches a resolution of approximately 500 after 28 cycles for the two peptides under the experimental conditions applied here. The instrument is not strictly limited to this resolution, since the number of cycles can be further increased, however doing so comes at the cost of having to decrease the $10 \mathrm{~Hz}$ repetition rate, because the separated ion packets start to overlap with freshly injected ones at the highest values of $n$. A practical limit is set by the currently observed loss of ions of up to $3 \%$ per cycle. An effort to further improve the ion transmission of the SLIM device is currently underway.

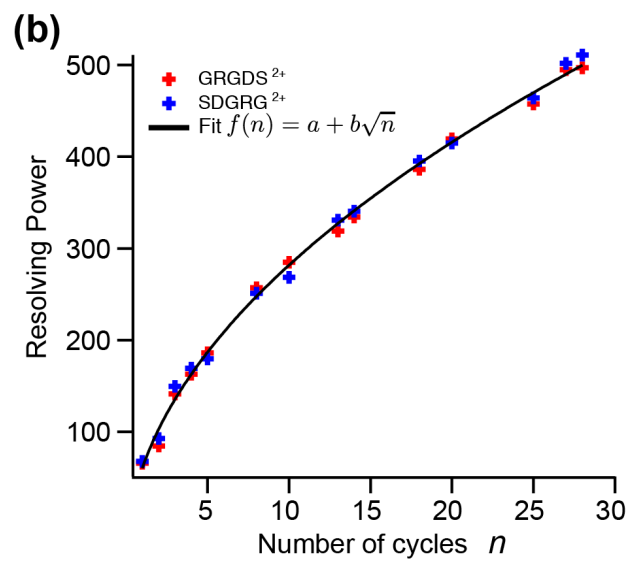

Figure 2 (a) Arrival time distribution from a mixture of isomeric peptides GRGDS and SDGRG (doubly protonated) are shown for different number of cycles $n$ (IMS path length). (b) IMS resolution of the new instrument as a function of the number of cycles $n$. 
Ion-mobility selective IR spectroscopy. While the combination of ion mobility spectrometry and cryogenic IR spectroscopy is promising for the identification of glycan isomers ${ }^{36,44}$ the resolution of standard IMS methods is a limiting factor. The goal of our approach is to separate glycan isomers as completely as possible before spectroscopic interrogation in a cryogenic ion trap to simplify the spectral deconvolution of any remaining unresolved species. We demonstrate here that the high resolution available using SLIM-based ion mobility $^{3940}$ is ideal for coupling with cryogenic IR spectroscopy for glycan analysis.

Figure 3(a) shows an ATD of the singly sodiated disaccharide Gal $\beta(1-4)$ GlcNAc measured on both our 2 m conventional drift tube IMS device ${ }^{45}$ (top panel) and in the newly developed cyclic SLIM instrument (second from top) for comparison. While in the $2 \mathrm{~m}$ drift tube only a single drift peak can be resolved, two well-separated species are apparent in the data from the cyclic IMS experiment after seven cycles, which corresponds to a $13 \mathrm{~m}$ drift path. At these experimental conditions, the resolution of the SLIM instrument reaches approximately 230 (Fig. 2(b)), which translates into a difference in collisional cross section of approximately $0.5 \%$ between the two separated species. These two species must represent either different conformations of the same isomer or different anomers at the reducing end ${ }^{41}$ (i.e., $\alpha$ or $\beta$ ). It is noteworthy that all reducing sugars that we have investigated show precisely two peaks after ion-mobility separation, which supports the hypothesis ${ }^{41}$ that the two separated conformers originate from the two anomers that are present in solution.

We now demonstrate the combination of our high-resolution ion-mobility separation with cryogenic IR spectroscopy. The $\mathrm{OH}$ and $\mathrm{NH}$ oscillators in carbohydrates provide excellent spectroscopic structural probes, because they are highly sensitive to local hydrogen bonding. We thus focus our infrared spectra on the wavenumber range between $3300 \mathrm{~cm}^{-1}$ and $3700 \mathrm{~cm}^{-1}$, where the corresponding stretching vibrations are expected. The vibrational spectrum of the disaccharide Galß(1-4)GlcNAc for which we do not select isomers after either the drift tube and or the SLIM device are displayed for comparison in the top two panels of Fig. 3(b), respectively. Absorption bands above $\sim 3580 \mathrm{~cm}^{-1}$ can be attributed to weakly hydrogen-bonded or free-OH oscillators, while more strongly hydrogen-bonded $\mathrm{OH}$ and $\mathrm{NH}$ oscillators appear at lower wavenumber. ${ }^{53}$ For the acquisition of the spectrum previously measured using our drift-tube apparatus, ${ }^{44}$ the ion trap temperature was set to $13 \mathrm{~K}$ and $\mathrm{D}_{2}$ was used as a messenger for photon absorption. In the current experiments using SLIM-based ion mobility separation, we maintain the trap at $40 \mathrm{~K}$ and use $\mathrm{N}_{2}$ as a messenger tag. Both the lower temperature and the weaker interaction of the $\mathrm{D}_{2}$ tag results in slightly narrower bands for the previously measured spectra. Except for this small difference in band width, the two spectra are almost identical in the number and position of the observed absorption bands, justifying the use of $\mathrm{N}_{2}$ tagging, which is experimentally less demanding. To ensure our spectra are reproducible, we perform all experiments under annealing conditions to yield the most stable gas-phase structures rather than kinetically trapped species. We have demonstrated the robustness of this approach by measuring the same IR spectrum months apart on the same instrument as well as on different instruments. ${ }^{54}$ This is an important prerequisite for the proposed approach to be employed as an analytical technique.

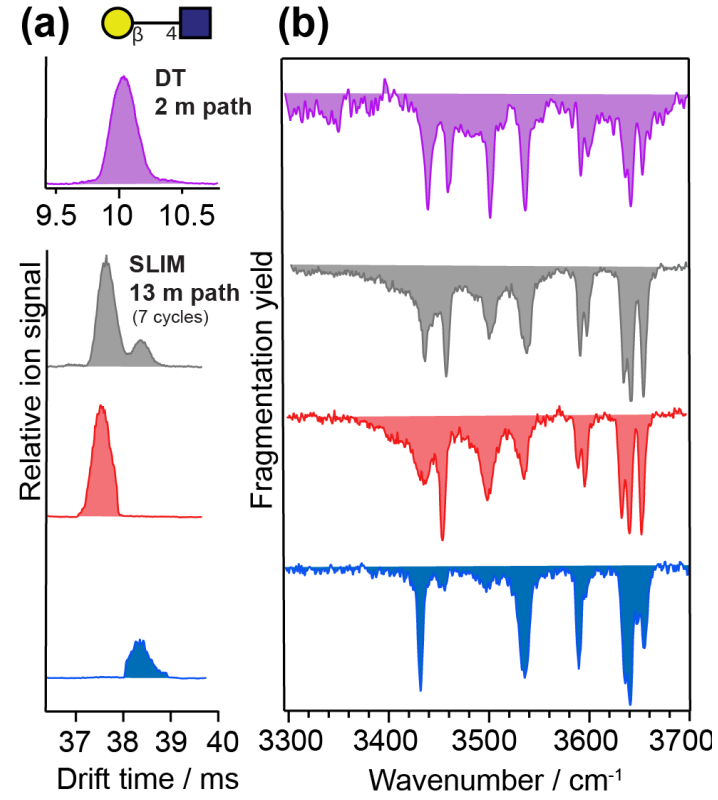

Figure 3 Comparison between $2 \mathrm{~m}$ drift tube (DT) and SLIM IMS resolution (a) Arrival time distributions (ATD) of the sodiated disaccharide Gal $\beta(1-4)$ GlcNAc obtained using the $2 \mathrm{~m}$ drift tube (top, purple), the new SLIM instrument (second from top, gray). The portions of the SLIM-separated ATD that was selected for spectroscopic analysis are shown in red and blue. (b) IR spectra of the IMS unseparated species from the drift-tube instrument (top, purple), and the new SLIM instrument (second from top, gray) for comparison and after mobility selecting of the first (red) and the second (blue) drift peaks using the SLIM instrument.

Using the separation capabilities of the SLIM-based IMS device, we can decompose the IR spectrum of Gal $\beta(1-4)$ GlcNAc into separate spectra for the two individual components (bottom two panels in Fig. 3(b)). It is evident that the spectra differ in band positions and intensities, which reflects the different intramolecular hydrogen-bonding network of two isomeric species. We demonstrate below that these IR fingerprint spectra can serve to unambiguously identify carbohydrate molecules from a mobility-separated mixture. ${ }^{44,55}$

Identification of glycans from mixtures. We test the capability of our new multidimensional ultrahigh-resolution IMS-cryogenic IR spectroscopy approach to distinguish and identify carbohydrate molecules in mixtures using a set of isomeric disaccharides as well as biologically relevant human milk oligosaccharides. For the former, we use the particularly challenging case of identification of epimers. We do this by comparing disaccharides with glucose versus galactose at the non-reducing end, yielding molecules that differ only in the orientation of the C-4 carbon (Fig. 4(a)).

Solutions of disaccharide epimers GalNAco(1-3)Gal and GlcNAc $\alpha(1-3) \mathrm{Gal})$ were first electrosprayed separately to record their IR spectra for reference. In both cases, two distinct drift peaks were observed for each molecule after several separation cycles, leading to two fingerprint IR spectra per glycan. These are displayed in Fig. 4(c) in red and blue for GlcNAc $\alpha(1-3)$ Gal (labeled I and II) and GalNAc $\alpha(1-3)$ Gal (labeled III and IV), respectively. These spectra can then be used for the identification of components in a mixture. Subsequently, a 1:1 mixture of the disaccharides was prepared and 
analyzed using our multidimensional approach. Figure 4(b) shows the ATD obtained from the mixture after eight cycles in the SLIM IMS region to reach a drift length of $15 \mathrm{~m}$, which corresponds to a resolving power of approximately 250 . Four distinct species (labeled 1 through 4) can be completely separated under these conditions and selected for spectroscopic analysis, and we show the resulting IR spectra in Fig. 4(c) (in grey).

Comparison of the spectra with reference spectra from the individually investigated compounds is required to test if the signals from the mixture can be assigned. Indeed, for each of the spectra 1 through 4 , a virtually identical reference spectrum can be found, which leads us to conclude that the first two drift peaks from the mixture (1 and 2) correspond to signals from GlcNAc $\alpha(1-3)$ Gal and the last two peaks (3 and 4) correspond to GalNAc $\alpha(1-3) \mathrm{Gal}$.

As a second demonstration of our approach, we use the human milk oligosaccharides lacto-N-tetraose (LNT) and lacto$\mathrm{N}$-neo-tetraose (LNnT). These tetrasaccharides are regioisomers that differ in the glycosidic linkage ( $\beta 1-3$ vs. $\beta 1-4)$ at the non-reducing end. As in the case of the disaccharides, ATDs of the individually electrosprayed, singly sodiated reference compounds each exhibit two distinct features (Fig. S2, Supporting Information). The reference IR spectra for these species are displayed in Fig. 5(c) in red and blue for the two drift peaks of LNnT (labeled I and II) and LNT (labeled III and
IV), respectively. Each spectrum shows several sharp transitions in the free-OH region above $3580 \mathrm{~cm}^{-1}$ and considerably broader absorption bands in the hydrogen-bonded region at lower wavenumber. Despite the fact that the spectra are generally more congested than those of the smaller disaccharides, they are still distinct and highly characteristic for a given structure of the molecule.

We then electrosprayed a 1:1 mixture of LNT and LNnT and observed three mobility-resolved species in the ATD after five cycles in the SLIM IMS section (labeled $\mathbf{1}$ through $\mathbf{3}$ in Fig. 5(b)). It is likely that the expected fourth component is simply not resolved under these experimental conditions. While the ion-mobility resolving power can be further increased using more separation cycles, this comes at the cost of the total number of ions transmitted to the ion trap. Nevertheless, IR spectra were recorded for the three individual ion mobility peaks from the mixture, displayed in Fig. 5(c) (grey) and labeled accordingly. As in the case of the disaccharide, it is straightforward to find a matching reference spectrum for each of the spectra 1,2 , and 3 by visual comparison and therefore to unambiguously determine the presence of both LNnT (peaks 1 and 2) and LNT (peak 3) in the mixture. The fact that the second component of LNT is not resolved here does not represent an obstacle for identification of the compound, because either one of the two reference IR spectra contain enough information to serve as a unique fingerprint.

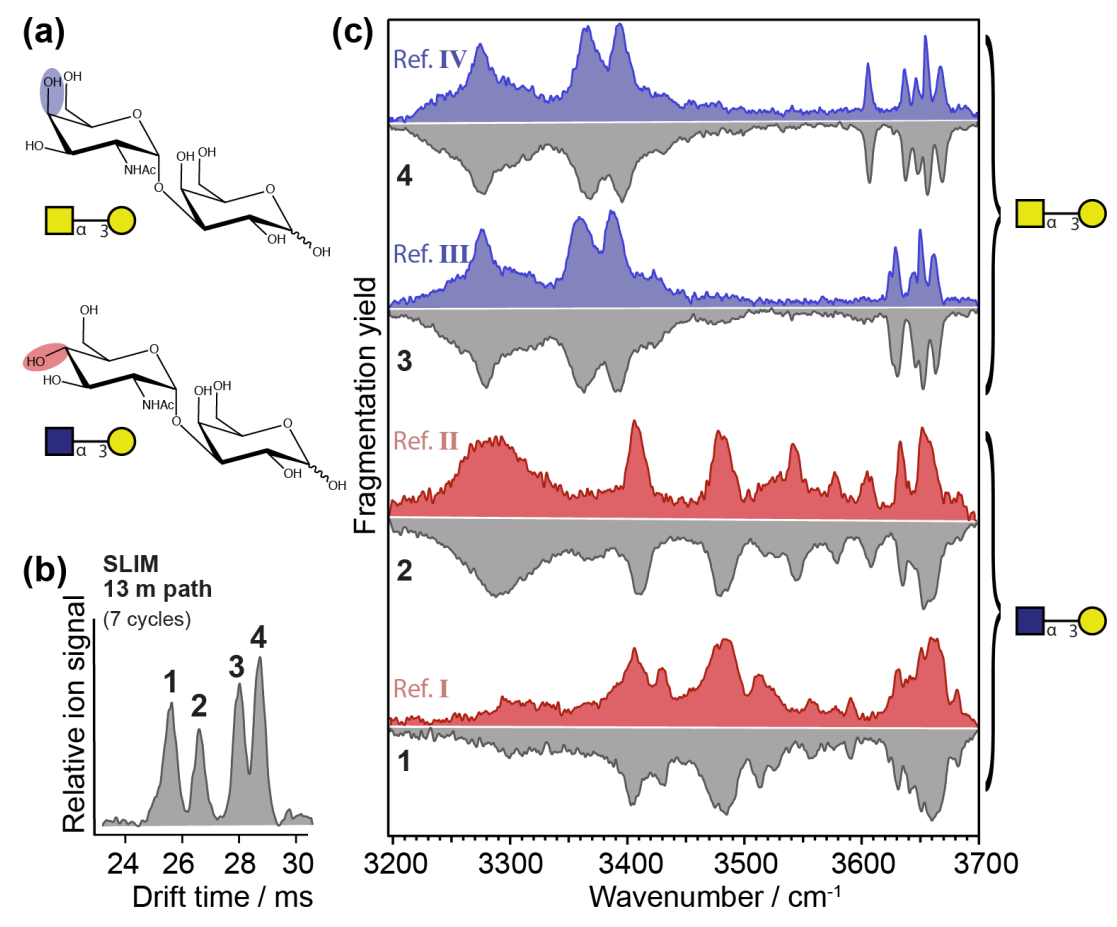

Figure 4 (a) Structures and Oxford notations of GalNAc $\alpha(1-3)$ Gal and GlcNAc $\alpha(1-3)$ Gal highlighting the subtle difference in structure between them. (b) Arrival time distribution of the mixture of the two sodiated disaccharides (c) IR spectra of the different nitrogen tagged, mobility-selected species form the mixture (gray) and obtained from individually investigated substances as references (red and blue). 


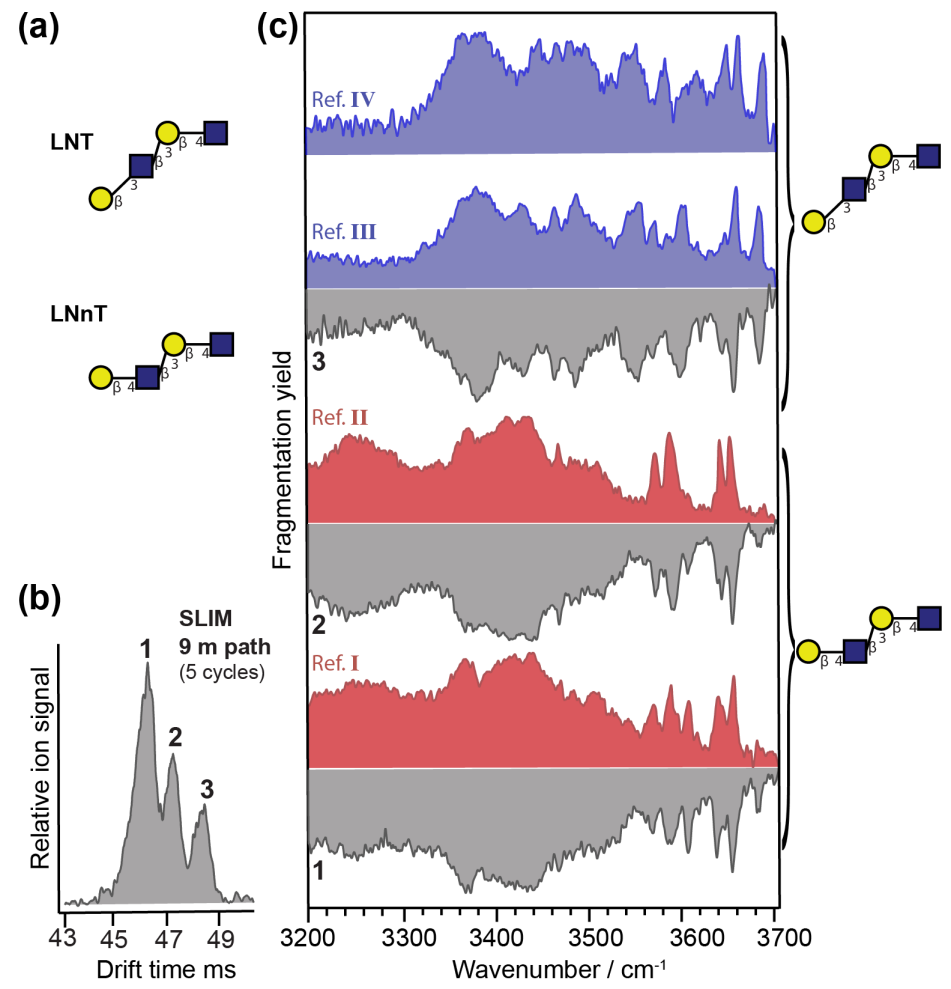

Figure 5 (a) Human milk tetrasaccharides LNT and LNnT in Oxford notation. (b) Arrival-time distribution of the mixture of sodiated LNT and LNnT. (c) ) IR spectra of the different nitrogen-tagged, mobility-selected species form the mixture (gray) and obtained from individually investigated substances as references (red and blue).

These results represent the first demonstration of using a database approach to unambiguously identify ion-mobility separated carbohydrates from a mixture on basis of their IR vibrational spectra. Two other examples where isomeric disaccharides are separated and identified from a mixture can be found in the Supporting Information (Figs. S3 and S4). While in the results reported here, spectra obtained from mixtures were compared and matched to reference spectra by simple visual inspection, in the future this process will be rapidly done by computer.

As demonstrated in the case of LNT and LNnT, the method is still easily applicable even when all gas-phase structures of one compound cannot be fully separated in the preparative IMS step. However, in a case where the ion-mobility resolving power will not be sufficient to separate different species, the resulting IR spectrum will simply be the sum of those from overlapping structures and can be deconvoluted using the reference database. Given the rapid development of ultrahighresolution ion-mobility instrumentation, we anticipate that such a deconvolution step will only be necessary in rare cases. The same technological development will also be beneficial for the analysis of larger glycan structures.

\section{CONCLUSIONS}

We demonstrate in this work that the combination of ultrahigh-resolution SLIM-based IMS-MS as a preparative step for messenger-tagging, cryogenic IR spectroscopy is a powerful technique for analyzing and distinguishing between isomeric glycans. The strength of this approach arises from the high degree of orthogonality between data from IMS-MS and cryogenic IR spectroscopy and their exquisite sensitivity to the finest details of glycan structure. Ongoing extensions of this method to operate at high throughput would profoundly impact the field of glycomics.

\section{ASSOCIATED CONTENT}

\section{Supporting Information}

The layout of the SLIM board is shown in detail. Additional arrival-time distributions (ATDs) for human milk oligosaccharides and cryogenic ion spectra and ATDs for two additional disaccharide mixtures are also included. The Supporting Information is available free of charge on the ACS Publications website.

\section{AUTHOR INFORMATION}

\section{Corresponding Author}

thomas.rizzo@epfl.ch

\section{ACKNOWLEDGMENT}

The authors thank the Swiss National Science Foundation (200020_165908), the European Research Council (788697GLYCANAL), and the EPFL for their generous support of this work. We would also like to acknowledge R.D. Smith and his group for helpful discussions on the implementation of the SLIM technology.

\section{REFERENCES}

1. Varki, A., Biological roles of glycans. Glycobiology 2017, 27 (1), 3-49.

2. Varki, A.; Cummings, R. D.; Esko, J. D.; Freeze, H. H.; Stanley, P.; Bertozzi, C. R.; Hart, G. W.; Etzler, M. E., Essentials of Glycobiology. 2nd ed.; Cold Spring Harbor Laboratory Press; 2009. : Cold Spring Harbor (NY), 2009.

3. Transforming Glycoscience: A Roadmap for the Future. The National Academies Press: Washington, D.C., 2012. 
4. Rudd, P. M.; Elliott, T.; Cresswell, P.; Wilson, I. A.; Dwek, R. A., Glycosylation and the immune system. Science 2001, 291 (5512), 2370-2376.

5. Laine, R. A., A calculation of all possible oligosaccharide isomers both branched and linear yields $1.05 \times 10(12)$ structures for a reducing hexasaccharide - The isomer-barrier to development of single-method saccharide sequencing or synthesis systems. Glycobiology 1994, 4 (6), 759-767.

6. Hofmann, J.; Stuckmann, A.; Crispin, M.; Harvey, D. J.; Pagel, K.; Struwe, W. B., Identification of Lewis and Blood Group Carbohydrate Epitopes by Ion Mobility-Tandem-Mass Spectrometry Fingerprinting. Anal. Chem. 2017, 89 (4), 2318-2325.

7. Harvey, D. J.; Struwe, W. B., Structural Studies of Fucosylated N-Glycans by Ion Mobility Mass Spectrometry and CollisionInduced Fragmentation of Negative Ions. J. Am. Soc. Mass Spectrom. 2018, 29 (6), 1179-1193.

8. Domon, B.; Costello, C. E., A systematic nomenclature for carbohydrate fragmentations in FAB-MS spectra of glycoconjugates Glycoconjugate Journal 1988, 5 (4), 397-409.

9. Khatri, K.; Pu, Y.; Klein, J. A.; Wei, J.; Costello, C. E.; Lin, C.; Zaia, J., Comparison of Collisional and Electron-Based Dissociation Modes for Middle-Down Analysis of Multiply Glycosylated Peptides. J. Am. Soc. Mass Spectrom. 2018, 29 (6), 1075-1085.

10. Adamson, J. T.; Håkansson, K., Electron Capture Dissociation of Oligosaccharides Ionized with Alkali, Alkaline Earth, and Transition Metals. Anal. Chem. 2007, 79 (7), 2901-2910.

11. Wolff, J. J.; Amster, I. J.; Chi, L.; Linhardt, R. J., Electron detachment dissociation of glycosaminoglycan tetrasaccharides. $J$. Am. Soc. Mass Spectrom. 2007, 18 (2), 234-244.

12. Zhao, C.; Xie, B.; Chan, S.-Y.; Costello, C. E.; O'Connor, P. B., Collisionally activated dissociation and electron capture dissociation provide complementary structural information for branched permethylated oligosaccharides. J. Am. Soc. Mass Spectrom. 2008, 19 (1), 138-150.

13. Yu, X.; Jiang, Y.; Chen, Y. J.; Huang, Y. Q.; Costello, C. E.; Lin, C., Detailed Glycan Structural Characterization by Electronic Excitation Dissociation. Anal. Chem. 2013, 85 (21), 10017-10021.

14. Tang, Y.; Pu, Y.; Gao, J. S.; Hong, P. Y.; Costello, C. E.; Lin, C., De Novo Glycan Sequencing by Electronic Excitation Dissociation and Fixed-Charge Derivatization. Anal. Chem. 2018, 90 (6), 3793 3801 .

15. Lin, Z.; Lubman, D. M., Permethylated N-Glycan Analysis with Mass Spectrometry. In Mass Spectrometry Data Analysis in Proteomics, Matthiesen, R., Ed. Humana Press: Totowa, NJ, 2013; pp 289-300.

16. Gray, C. J.; Thomas, B.; Upton, R.; Migas, L. G.; Eyers, C. E.; Barran, P. E.; Flitsch, S. L., Applications of ion mobility mass spectrometry for high throughput, high resolution glycan analysis. Biochim Biophys Acta 2016, 1860 (8), 1688-709.

17. Gaye, M. M.; Nagy, G.; Clemmer, D. E.; Pohl, N. L. B., Multidimensional Analysis of 16 Glucose Isomers by Ion Mobility Spectrometry. Anal. Chem. 2016, 88 (4), 2335-2344.

18. Hofmann, J.; Hahm, H. S.; Seeberger, P. H.; Pagel, K., Identification of carbohydrate anomers using ion mobility-mass spectrometry. Nature 2015, 526 (7572), 214-244.

19. Gaye, M. M.; Kurulugama, R.; Clemmer, D. E., Investigating carbohydrate isomers by IMS-CID-IMS-MS: precursor and fragment ion cross-sections. Analyst 2015, 140 (20), 6922-6932.

20. Pagel, K.; Harvey, D. J., Ion Mobility-Mass Spectrometry of Complex Carbohydrates: Collision Cross Sections of Sodiated Nlinked Glycans. Anal. Chem. 2013, 85 (10), 5138-5145.

21. Guttman, M.; Lee, K. K., Site-Specific Mapping of Sialic Acid Linkage Isomers by Ion Mobility Spectrometry. Anal. Chem. 2016, 88 (10), 5212-5217.

22. Both, P.; Green, A. P.; Gray, C. J.; ŠardzíkR; VoglmeirJ; FontanaC; AusteriM; RejzekM; RichardsonD; Field, R. A.; WidmalmG; Flitsch, S. L.; Eyers, C. E., Discrimination of epimeric glycans and glycopeptides using IM-MS and its potential for carbohydrate sequencing. Nat Chem 2014, 6 (1), 65-74.

23. Chen, Z.; Glover, M. S.; Li, L., Recent advances in ion mobilitymass spectrometry for improved structural characterization of glycans and glycoconjugates. Current Opinion in Chemical Biology 2018, $42,1-8$.

24. Pu, Y.; Ridgeway, M. E.; Glaskin, R. S.; Park, M. A.; Costello, C. E.; Lin, C., Separation and Identification of Isomeric Glycans by Selected Accumulation-Trapped Ion Mobility Spectrometry-Electron Activated Dissociation Tandem Mass Spectrometry. Anal. Chem. 2016, 88 (7), 3440-3443.

25. Harvey, D. J.; Watanabe, Y.; Allen, J. D.; Rudd, P.; Pagel, K.; Crispin, M.; Struwe, W. B., Collision Cross Sections and Ion Mobility Separation of Fragment Ions from Complex N-Glycans. $J$. Am. Soc. Mass Spectrom. 2018, 29 (6), 1250-1261.

26. Struwe, W. B.; Pagel, K.; Benesch, J. L. P.; Harvey, D. J.; Campbell, M. P., GlycoMob: an ion mobility-mass spectrometry collision cross section database for glycomics. Glycoconjugate Journal 2016, 33 (3), 399-404.

27. Polfer, N. C.; Valle, J. J.; Moore, D. T.; Oomens, J.; Eyler, J. R.; Bendiak, B., Differentiation of isomers by wavelength-tunable infrared multiple-photon dissociation-mass spectrometry: Application to glucose-containing disaccharides. Anal. Chem. 2006, 78 (3), 670-679.

28. Hernandez, O.; Isenberg, S.; Steinmetz, V.; Glish, G. L.; Maitre, P., Probing Mobility-Selected Saccharide Isomers: Selective IonMolecule Reactions and Wavelength-Specific IR Activation. J. Phys. Chem. A 2015, 119 (23), 6057-6064.

29. Tan, Y. L.; Polfer, N. C., Linkage and Anomeric Differentiation in Trisaccharides by Sequential Fragmentation and VariableWavelength Infrared Photodissociation. J. Am. Soc. Mass Spectrom. 2015, 26 (2), 359-368.

30. Gray, C. J.; Schindler, B.; Migas, L. G.; Pičmanová, M.; Allouche, A. R.; Green, A. P.; Mandal, S.; Motawia, M. S.; SánchezPérez, R.; Bjarnholt, N.; Møller, B. L.; Rijs, A. M.; Barran, P. E.; Compagnon, I.; Eyers, C. E.; Flitsch, S. L., Bottom-Up Elucidation of Glycosidic Bond Stereochemistry. Anal. Chem. 2017, 89 (8), 4540-4549.

31. Schindler, B.; Barnes, L.; Gray, C. J.; Chambert, S.; Flitsch, S. L.; Oomens, J.; Daniel, R.; Allouche, A. R.; Compagnon, I., IRMPD Spectroscopy Sheds New (Infrared) Light on the Sulfate Pattern of Carbohydrates. J. Phys. Chem. A 2017, 121 (10), 2114-2120.

32. Schindler, B.; Renois-Predelus, G.; Bagdadi, N.; Melizi, S.; Barnes, L.; Chambert, S.; Allouche, A.-R.; Compagnon, I., MS/IR, a new MS-based hyphenated method for analysis of hexuronic acid epimers in glycosaminoglycans. Glycoconjugate Journal 2017, 34 (3), 421-425.

33. Mucha, E.; González Flórez, A. I.; Marianski, M.; Thomas, D. A.; Hoffmann, W.; Struwe, W. B.; Hahm, H. S.; Gewinner, S.; Schöllkopf, W.; Seeberger, P. H.; von Helden, G.; Pagel, K., Glycan Fingerprinting using Cold-Ion Infrared Spectroscopy. Angew. Chem. Int. Ed. 2017, n/a-n/a.

34. Schindler, B.; Barnes, L.; Renois, G.; Gray, C.; Chambert, S.; Fort, S.; Flitsch, S.; Loison, C.; Allouche, A.-R.; Compagnon, I., Anomeric memory of the glycosidic bond upon fragmentation and its consequences for carbohydrate sequencing. Nature Communications 2017, 8 .

35. Schindler, B.; Laloy-Borgna, G.; Barnes, L.; Allouche, A.-R.; Bouju, E.; Dugas, V.; Demesmay, C.; Compagnon, I., Online separation and identification of isomers using IRMPD ion spectroscopy coupled to liquid chromatography: application to the analysis of disaccharides regio-isomers and monosaccharide anomers. Anal. Chem. 2018.

36. Khanal, N.; Masellis, C.; Kamrath, M. Z.; Clemmer, D. E.; Rizzo, T. R., Cryogenic IR spectroscopy combined with ion mobility spectrometry for the analysis of human milk oligosaccharides. Analyst 2018, 143 (8), 1846-1852.

37. Masellis, C.; Khanal, N.; Kamrath, M. Z.; Clemmer, D. E.; Rizzo, T. R., Cryogenic Vibrational Spectroscopy Provides Unique Fingerprints for Glycan Identification. J. Am. Soc. Mass Spectrom. 2017, 28, 2217-2222.

38. Khanal, N.; Masellis, C.; Kamrath, M. Z.; Clemmer, D. E.; Rizzo, T. R., Glycosaminoglycan analysis by cryogenic messengertagging IR spectroscopy combined with IMS-MS. Anal. Chem. 2017, 89, 7601-7606. 
39. Deng, L.; Ibrahim, Y. M.; Hamid, A. M.; Garimella, S. V.; Webb, I. K.; Zheng, X.; Prost, S. A.; Sandoval, J. A.; Norheim, R. V.; Anderson, G. A.; Tolmachev, A. V.; Baker, E. S.; Smith, R. D., UltraHigh Resolution Ion Mobility Separations Utilizing Traveling Waves in a $13 \mathrm{~m}$ Serpentine Path Length Structures for Lossless Ion Manipulations Module. Anal Chem 2016, 88 (18), 8957-64.

40. Hamid, A. M.; Garimella, S. V. B.; Ibrahim, Y. M.; Deng, L.; Zheng, X.; Webb, I. K.; Anderson, G. A.; Prost, S. A.; Norheim, R. V.; Tolmachev, A. V.; Baker, E. S.; Smith, R. D., Achieving High Resolution Ion Mobility Separations Using Traveling Waves in Compact Multiturn Structures for Lossless Ion Manipulations. Anal. Chem. 2016, 88 (18), 8949-8956.

41. Nagy, G.; Attah, I. K.; Garimella, S. V. B.; Tang, K.; Ibrahim, Y. M.; Baker, E. S.; Smith, R. D., Unraveling the isomeric heterogeneity of glycans: ion mobility separations in structures for lossless ion manipulations. Chem Commun (Camb) 2018, 54 (83), 11701-11704. 42. Lorenz, U. J.; Rizzo, T. R., Planar multipole ion trap/time-offlight mass spectrometer. Anal Chem 2011, 83 (20), 7895-901.

43. Kamrath, M. Z.; Rizzo, T. R., Combining Ion Mobility and Cryogenic Spectroscopy for Structural and Analytical Studies of Biomolecular Ions. Accounts of Chemical Research 2018, 51 (6), 1487-1495.

44. Masellis, C.; Khanal, N.; Kamrath, M. Z.; Clemmer, D. E.; Rizzo, T. R., Cryogenic Vibrational Spectroscopy Provides Unique Fingerprints for Glycan Identification. J Am Soc Mass Spectrom 2017, 28 (10), 2217-2222.

45. Masson, A.; Kamrath, M. Z.; Perez, M. A.; Glover, M. S.; Rothlisberger, U.; Clemmer, D. E.; Rizzo, T. R., Infrared Spectroscopy of Mobility-Selected H+-Gly-Pro-Gly-Gly (GPGG). $J$ Am Soc Mass Spectrom 2015, 26 (9), 1444-54.

46. Pauly, M.; Sroka, M.; Reiss, J.; Rinke, G.; Albarghash, A.; Vogelgesang, R.; Hahne, H.; Kuster, B.; Sesterhenn, J.; Kern, K.; Rauschenbach, S., A hydrodynamically optimized nano-electrospray ionization source and vacuum interface. Analyst 2014, 139 (8), 185667.

47. Ibrahim, Y.; Belov, M. E.; Tolmachev, A. V.; Prior, D. C.; Smith, R. D., Ion funnel trap interface for orthogonal time-of-flight mass spectrometry. Anal Chem 2007, 79 (20), 7845-52.

48. Ibrahim, Y. M.; Hamid, A. M.; Deng, L.; Garimella, S. V.; Webb, I. K.; Baker, E. S.; Smith, R. D., New frontiers for mass spectrometry based upon structures for lossless ion manipulations. Analyst 2017, 142 (7), 1010-1021.

49. Garimella, S. V.; Ibrahim, Y. M.; Webb, I. K.; Ipsen, A. B.; Chen, T. C.; Tolmachev, A. V.; Baker, E. S.; Anderson, G. A.; Smith, R. D., Ion manipulations in structures for lossless ion manipulations (SLIM): computational evaluation of a 90 degrees turn and a switch. Analyst 2015, 140 (20), 6845-52.

50. Shvartsburg, A. A.; Smith, R. D., Fundamentals of Traveling Wave Ion Mobility Spectrometry. Anal. Chem. 2008, 80 (24), 96899699.

51. Gordon, S. D. S.; Osterwalder, A., 3D-Printed Beam Splitter for Polar Neutral Molecules. Physical Review Applied 2017, 7 (4).

52. Kemper, P. R.; Dupuis, N. F.; Bowers, M. T., A new, higher resolution, ion mobility mass spectrometer. International Journal of Mass Spectrometry 2009, 287 (1-3), 46-57.

53. Voss, J. M.; Kregel, S. J.; Fischer, K. C.; Garand, E., IR-IR Conformation Specific Spectroscopy of $\mathrm{Na}+($ Glucose) Adducts. $J$. Am. Soc. Mass Spectrom. 2018, 29 (1), 42-50.

54. Warnke, S.; Ben Faleh, A.; Pellegrinelli, R. P.; Yalovenko, N.; Rizzo, T. R., Combining ultra-high resolution ion-mobility spectrometry with cryogenic IR spectroscopy for the study of biomolecular ions. Faraday Discussions 2018.

55. Mucha, E.; Stuckmann, A.; Marianski, M.; Struwe, W. B.; Meijer, G.; Pagel, K., In-depth structural analysis of glycans in the gas phase. Chemical Science 2019.

56. Hoffmann, W.; Hofmann, J.; Pagel, K., Energy-resolved ion mobility-mass spectrometry--a concept to improve the separation of isomeric carbohydrates. J Am Soc Mass Spectrom 2014, 25 (3), 4719. 